\title{
Symmetry Modes and Stiffnesses for Bimanual Rehabilitation
}

\author{
Samuel McAmis and Kyle B. Reed \\ Department of Mechanical Engineering \\ University of South Florida \\ Tampa, Florida, 33612 \\ Email: smcamis@mail.usf.edu, kylereed@usf.edu
}

\begin{abstract}
Bimanual rehabilitation devices show promise for use in low cost trainers for home use. To gain a better understanding of the symmetry modes and coupling stiffnesses that would be beneficial for home use bimanual trainers, we conducted a haptic tracking task. Participants used one hand to recreate the trajectory applied by a robot to the other hand using three bimanual symmetry modes. The participants recreated visual symmetry and joint space (mirror) symmetry more easily than point mirror symmetry. Joint space symmetry was the easiest mode when the trajectory was an increasing chirp frequency function. The stiffness between the robot and one hand affected the coordination between both hands and stiffnesses of $200-700 \mathrm{~N} / \mathrm{m}$ enabled better tracking than $50 \mathrm{~N} / \mathrm{m}$.
\end{abstract}

\section{INTRODUCTION}

The symmetry of the human body allows for easy duplication of the motions on the left and right sides. Whereas it is difficult to pat ones head and rub ones stomach at the same time, it is very easy to draw circles rotating in either the same or opposite direction. Whether the direction of the circles is the same or opposite represents the frame of reference that the symmetry is in. Since a stroke typically impairs one side of the body while the other side is largely unaffected, the idea of bimanual rehabilitation is to physically couple the individual's two limbs such that the healthy limb can guide the impaired limb. Symmetric motor tasks could allow individuals with a stroke to self-rehabilitate at home by using their healthy arm to guide the motions of their affected arm. Self-rehabilitation is ideal for home-use since much of the required force could be provided by the patient's healthy limb instead of the larger motors included on many current upper limb rehabilitation robots. Our goal in this work is to identify the ideal bilateral interactions necessary for the external physical coupling between the two arms for bimanual rehabilitation. The research presented in this paper examines which symmetric patterns and coupling stiffness are likely to be the most effective for self-rehabilitation.

\section{BACKGROUND}

The goal of upper limb rehabilitation following a stroke is to enable a person to use both hands in activities of daily living. There have been many new rehabilitation methods proposed and tested in recent years. Many of these methods found in the rehabilitation literature show positive results, but there is no method that clearly shows better results than traditional methods [1][2]. A common thread among all these successful studies is that the amount of time spent training the affected arm plays an important role in improving the functional ability of the affected arm. As it is difficult for therapists to devote as much time as is needed, researchers have looked to robotic and other methods to supplement rehabilitation.

\section{A. Rehabilitation Techniques}

For nearly a hundred years, constraint-induced movement therapy has been the standard for stroke rehabilitation [3]. Sometimes referred to as forced non-use or forced use of the paretic limb, this method involves binding an individual's healthy limb so he is forced to use his paretic limb in everyday tasks. This method has the dual advantages of allowing the person to train for extensive periods of time and forcing the person to plan and execute motions relevant to everyday life. Unfortunately, because the individual must accomplish these tasks without the aid of their healthy arm, this method is only viable in individuals with small to moderate impairment.

A large literature of research detailing robotic methods for upper limb rehabilitation has come about in the past 15 years. A significant amount of this robotic rehabilitation methods focus on rehabilitating the impaired limb in planar tasks separate from the healthy hand. These methods can be divided into two types: assistive forces and resistive forces. Recent review papers [1][2] have stated that robotic training methods performed similarly to other upper extremity training methods when used for the same amount of time and that it is unclear whether robotic methods have the potential to produce greater benefits than conventional techniques. However, the advantage of these devices comes from the fact that the patients can use them for longer and more frequent periods of time in the clinic. The literature has shown that the amount of training is one of the most important factors for functional recovery after a stroke [4][5]. Given that robotic devices are expensive, complex, and potentially dangerous, the ideal option for homeuse would likely be a safe, affordable, and readily available method that is capable of generating the desired rehabilitation.

To allow patients greater access to rehabilitative training, several methods have been developed to allow patients to rehabilitate at home. One home based method is UniTherapy, which uses a force feedback joystick and steering wheel [6][7] and has been validated in clinical trials [8][9]. Another is Java Therapy, which uses a standard computer with internet access and can interface with a variety of force feedback devices [10]. Many of these home-based methods, however, use a home computer with limited accessories that cannot provide assistance forces and can only operate over a small workspace. These methods are able to provide some benefit, but the rehabilitation effect is limited to people who have relatively high motor function. 


\section{B. Bimanual Rehabilitation}

The fundamental concept of bimanual rehabilitation is that an individual uses their healthy arm to assist their own impaired arm through the use of an external coupling. The key mechanism of rehabilitation is that the same neural signal is sent to the arms, which will result in the same proprioceptive feedback from each limb since the arms are constrained to move together. Sending the same efferent signals to each limb will result in similar afferent signals from the limbs, which will help retrain the motor pathways to the impaired side [11][12]. Several research groups have studied certain aspects of bimanual rehabilitation, but few studies to date have examined what the ideal physical parameters for bimanual interaction should be.

One system, the Mirror Image Movement Enabler (MIME), uses a large industrial robot, the PUMA 560, to move the impaired arm in a mirror like fashion [13]. Their results show rehabilitative effects similar to many other current methods. The BiManuTrack also works to mirror the movements of the two limbs and has shown positive results similar to the MIME [14]. The BATRAC uses a similar mirror motion where the individuals move their hands on independent tracks, but the device does not provide any assistance [15], thus limiting the use to relatively high functioning individuals with stroke.

It is not currently known which types of symmetry modes are most effective for bimanual rehabilitation. Mirror motions are biomechanically interesting because the efferent signals can be duplicated at a low level since the joints on each limb are identical. Thus, mirror motions have been the most commonly used in bimanual rehabilitation studies to date. However, most daily tasks occur in a visual reference frame where the hands move in the same direction. One training program simulated a driving task that uses both arms simultaneously to reduce the effects of learned non-use of the impaired arm [8]. The research presented here compares bimanual motions in three common reference frames: Mirror or Joint Space Symmetry (JSS), Visual Symmetry (VS), and Point Mirror Symmetry (PMS), as shown in Fig. 1.

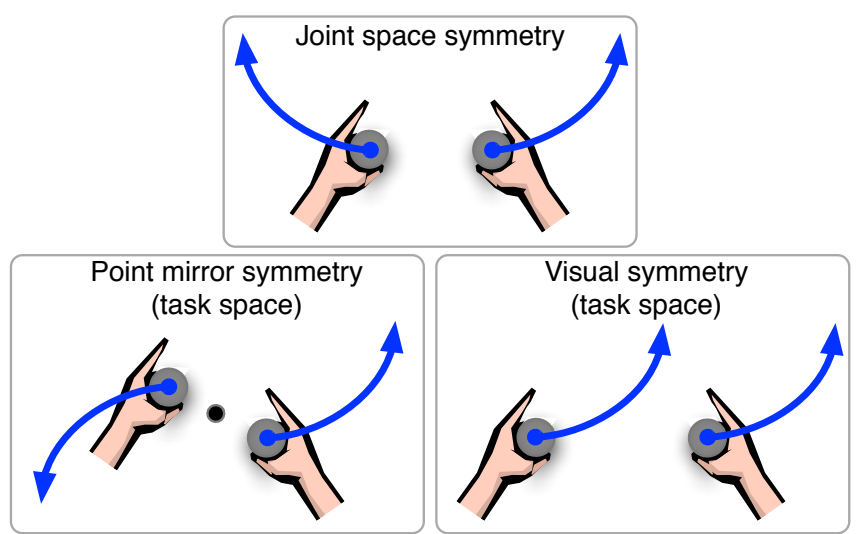

Fig. 1. The Bimanual symmetry modes tested here consist of Joint Space Symmetry (JSS), where the joint angles are mirrored, Visual Symmetry (VS), where the hands move through the same visual path, and Point Mirror Symmetry (PMS), where the hand motions are mirrored about a single point.
Another question that has received little attention deals with the compliance of the physical coupling between the hands. All of the above studies either did not physically connect the hands or coupled the hands rigidly. The ideal coupling is likely somewhere in between, since a soft coupling would prevent severely impaired individuals from using this training method and, with a completely rigid connection, the individual is likely to apply minimal force in their impaired hand since the healthy side will dictate all the motions [1][16]. The study reported here examines the effect of stiffness on a bimanual task with the long term goal of determining the beneficial range for bimanual rehabilitation trainers.

An initial study of Bimanual symmetric motions on healthy participants tested VS and JSS coupling with eight different paths at one stiffness level [17]. That study showed that VS tasks tended to be easier to perform than JSS. It was also shown that most participants could recreate slow, 0.5 and $1 \mathrm{~Hz}$, simple harmonic frequencies easily. The performance on superimposed harmonic frequencies, $0.5 \& 1.0 \mathrm{~Hz}$ and $0.5 \& 1.5 \mathrm{~Hz}$, was only slightly more difficult. Similar speed non-harmonic combinations of frequencies such as $0.7 \& 1.1 \mathrm{~Hz}$ proved difficult, but not impossible to follow. Both the previous study and the results presented here focus on healthy individuals in order to obtain a baseline measure and impaired individuals will be tested at a later time using a device designed based on the results of this study.

\section{Bimanual TRACKING}

The objective of this study was to evaluate individuals' ability to recreate a given motion with one hand as a robot guides their other hand. The three symmetry modes, shown in Fig. 1, and four spring stiffnesses were tested. The spring constants chosen were $50 \mathrm{~N} / \mathrm{m}, 200 \mathrm{~N} / \mathrm{m}, 500 \mathrm{~N} / \mathrm{m}$ and $700 \mathrm{~N} / \mathrm{m}$. The upper limit of the spring constants was set by the limitations of the Phantom Omni devices used to provide the guiding motion and the lower limit was set to be a very weak guiding motion.

\section{A. Procedure}

Participants sat in front of two Phantom Omni force feedback devices and held an Omni stylus in each hand. To maximize the range of forces that the Omnis could provide, they were positioned facing the participant for JSS and VS, and back to back with one Omni facing to the left and one to the right for PMS as shown in Fig. 2. The input Omni interacting with the participant's right hand applied a force that guided their hand through the desired trajectory. This trajectory consisted of either a single, double, or triple superimposed sine wave, or a chirp frequency. The force was applied based on the difference between the desired and actual hand positions: $F=k *\left(x_{\text {desired }}-x_{\text {measured }}\right)$, where $k$ is the spring constant. The output Omni interacting with the participant's left hand only measured the recreated trajectory and did not provide any force. Participants were instructed to simultaneously recreate the path applied to their right hand with their left hand. 


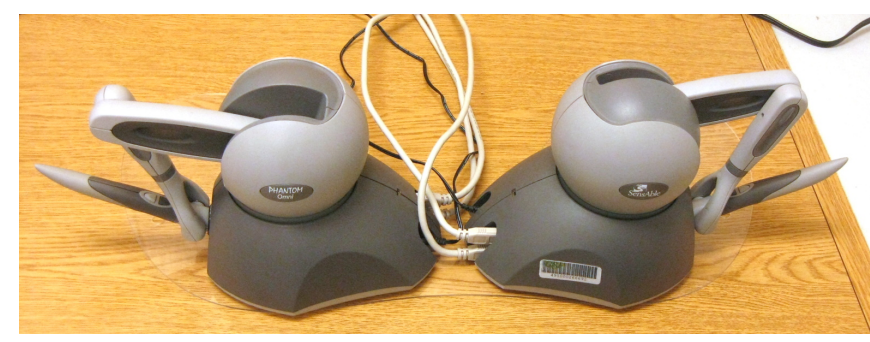

Fig. 2. Phantom Omnis arranged for Point Mirror Symmetry.

Eight participants performed this experiment: three females and five males, age $21-24$. Seven participants were right handed, one was left handed. None of the individuals tested had any impairment that would limit their motion. The study was approved by the University of South Florida's IRB.

Four input trajectories were tested: a single frequency sine wave of $0.5 \mathrm{~Hz}$, two superimposed frequencies of $0.9 \& 1.3 \mathrm{~Hz}$, three superimposed frequencies of $0.6,0.8, \& 1.1 \mathrm{~Hz}$, and a chirp frequency that increased linearly in frequency from 0 to $2.4 \mathrm{~Hz}$ over the course of the trial. The fixed frequencies were chosen to provide a range of difficulties, and the superimposed frequencies were selected to provide a pseudo-random path for the participant so that it would be more difficult to predict the trajectory. Each trial lasted 23 seconds with an initial 1.5 second ramp up period.

Each unique combination of symmetry type, spring constant, and trajectory was tested once with the exception of the chirp frequency, which was tested twice for each combination of symmetry type and spring constant. The order of each combination of spring constant and trajectory and the order of the symmetry types was randomized. However, to avoid confusion, all of the combinations of spring constants and trajectories for a given symmetry type were tested before moving to the next symmetry type. Participants were instructed to take a short break before starting each symmetry type.

\section{B. Data Analysis}

We performed a Fast Fourier transform to analyze the motions of the fixed frequency trials. For consistent data analysis the JSS motions were flipped $(x=-x)$ so the positions would be directly comparable to the input. Several metrics were used to determine how well the participant followed the given path: Power Score, Noise, and Total Lag. First, the Power Score was determined according to

$$
S= \begin{cases}0, & P<0.2 * I \\ \frac{P / I-0.2}{0.7}, & 0.2 * I<P<0.9 * I \\ 1, & 0.9 * I<P<1.1 * I \\ \frac{1.1-P / I}{1.8}+1, & 1.1 * I<P<2 I \\ 0.5, & P>2 I,\end{cases}
$$

where $S$ is the Power Score, $P$ is the output power, and $I$ is input power. If the power of the output frequency was within $10 \%$ of the input power, the participant was successfully following and awarded a score of 1 . If the output power was less than $20 \%$ of the input power, the participant was considered to not be following that frequency, and awarded a score of 0 . The participant's score was scaled linearly between these points. The participant's score was penalized for producing a larger amplitude response than the input, varying linearly from a score of 1 at $110 \%$ to 0.5 at $200 \%$. We used this scaling to determine the score so that neither random motions nor motions larger than the input would get weighted too heavily.

The Noise was calculated as the average power of the output frequencies that did not correspond to one of the input frequencies. The Total Lag was calculated by finding the lag at which the correlation was maximum between the input and output paths. An example plot and metrics are shown in Fig. 3.

To determine how well participants were following the chirp frequency, the Total Lag and the Average Lag were used. The Total Lag was calculated the same as it was for the constant frequency paths. The lag as a function of time was calculated using a window two periods in width centered about the point at which the lag was being determined. A representative lag vs. time graph and metrics are shown in Fig. 4. The average of the absolute value of these lags was calculated to determine the Average Lag for each trial. The absolute value was used to eliminate the participant receiving credit for producing negative lags as a result of lagging enough to be leading the next motion as occurs at approximately 21 seconds in Fig. 4. Based on the plots, the maximum frequency that participants were able to attain was typically in the 1.6 to $2.4 \mathrm{~Hz}$ range.

For our primary analysis, we compared the fixed and chirp frequency metrics of the desired path of the guided hand to the recreated path. We also compared the desired path to the actual path of the guided hand, and the actual path of the guided hand to the recreated path to determine if either contributed disproportionately to the total difference.

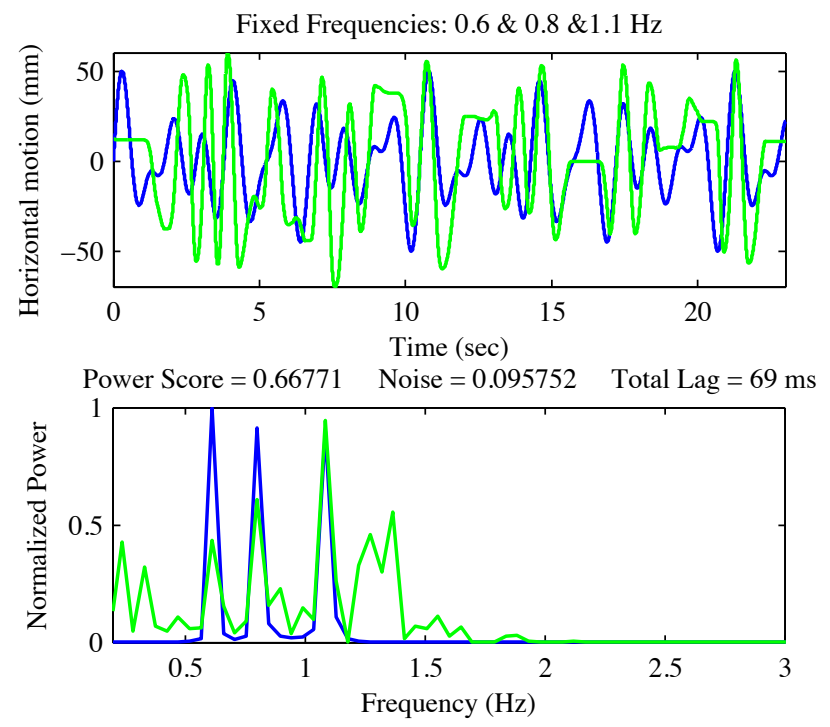

Fig. 3. Example of fixed frequency analysis plot and metrics. The first plot shows the desired path (dark blue), and the participant's recreated path (light green). The second plot shows a Fast Fourier Transform of the data. The performance metrics are shown in between. 

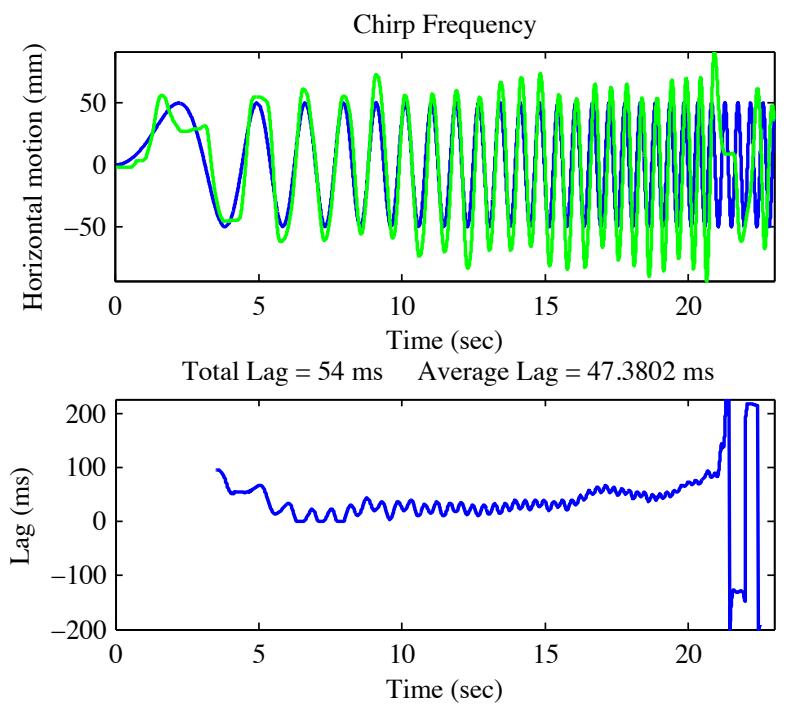

Fig. 4. Example of chirp frequency analysis plot and metrics. The top plot shows the desired path (dark blue), and the participant's recreated path (light green). The second plot shows the lag as a function of time over the course of the trial. The performance metrics are shown in between.

\section{Results}

We conducted a one-way analysis of variance (ANOVA) to analyze the effects of virtual spring stiffness, symmetry mode, and input frequency on the Power Score, Noise, Total Lag, and Average Lag. When the ANOVA yielded significant results, we used Turkey's honest significant difference test. We used an alpha of 0.05 for all statistical tests.

For the fixed frequencies the Power Score at the driven frequencies produced statistically significant results for distinguishing between symmetries $\left(p<10^{-4}\right)$, between virtual spring stiffnesses $\left(p<10^{-4}\right)$, and between trajectory paths $\left(p<10^{-4}\right)$. The results of the Power Score analysis are shown in Fig. 5. A higher Power Score indicates that the participant is recreating the input trajectory more accurately. Post hoc analyses showed that the participants had significantly more difficulty reproducing the motion in the PMS symmetry mode than the other two. This was in agreement with what the majority of participants verbally reported regarding the difficulty of each symmetry set. It is also seen that there is a strong overlap of the performance in JSS and VS. Learning effects were present in the Power Score $(P=0.0127)$, but there was no discernible pattern relating to any of the changes in symmetry type, stiffness, or trajectory.

Post hoc analysis of the Power Score for different stiffnesses showed that the $50 \mathrm{~N} / \mathrm{m}$ stiffness produced poorer tracking than other virtual spring stiffnesses. However, the other stiffnesses all produced similar results. Unsurprisingly, it was seen that participants had more difficulty following the desired path with their guided hand at this lower stiffness.

Post hoc analysis of the Power Score for the different trajectory paths showed that the single $0.5 \mathrm{~Hz}$ frequency trajectory was the easiest to reproduce, followed by the triple superimposed frequencies of $0.6,0.8 \& 1.1$ and then the double superimposed frequencies of $0.9 \& 1.3 \mathrm{~Hz}$. The Noise and
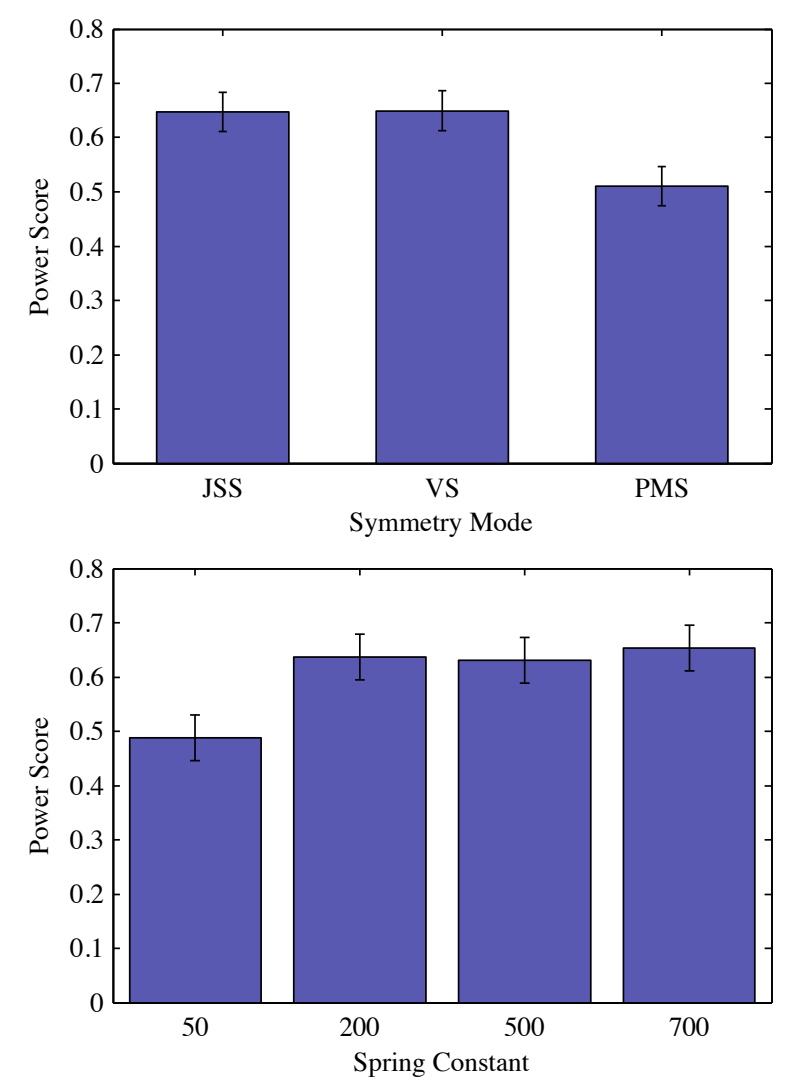

Fig. 5. Results of Power Score analysis between desired and recreated paths for fixed frequencies. A high Power Score indicates better tracking. Error bars represent $95 \%$ confidence interval.

Total Lag also produced statistically significant results $(p<$ $10^{-4}$ ) for the fixed frequencies. The Noise, while showing the single frequency trajectory as the easiest to produce, also showed that fewer extraneous frequencies were created when following the trajectory with two superimposed frequencies than with three superimposed frequencies.

Post hoc analysis of the Total Lag shows similar results to the analysis of the Power Score; the participants recreated the single frequency path while lagging less than the superimposed frequencies. As shown in Fig. 6, the mean Total Lag is negative for the single $0.5 \mathrm{~Hz}$ frequency, which indicates that, on average, the participants were leading the input trajectory. This is likely a result of the participants attempting to predict the motions, rather than sensing them.

The stiffness of the guiding force from the Omni affects the coordination between the participants two hands. The lag between the participants' actual path and the desired path was larger for the the $50 \mathrm{~N} / \mathrm{m}$ stiffness than for the other three stiffnesses. However, analysis of the Total Lag between the actual path of the guided hand and the recreated path also showed statistically significant results $(p<0.001)$. Post hoc analysis of the Total Lag between the actual path of the guided hand and the recreated path at different stiffnesses demonstrated a higher Total Lag for the $50 \mathrm{~N} / \mathrm{m}$ stiffness than the $500 \mathrm{~N} / \mathrm{m}$ stiffness or the $700 \mathrm{~N} / \mathrm{m}$ stiffness, as seen in Fig. 7. This result is particularly interesting because it indicates 


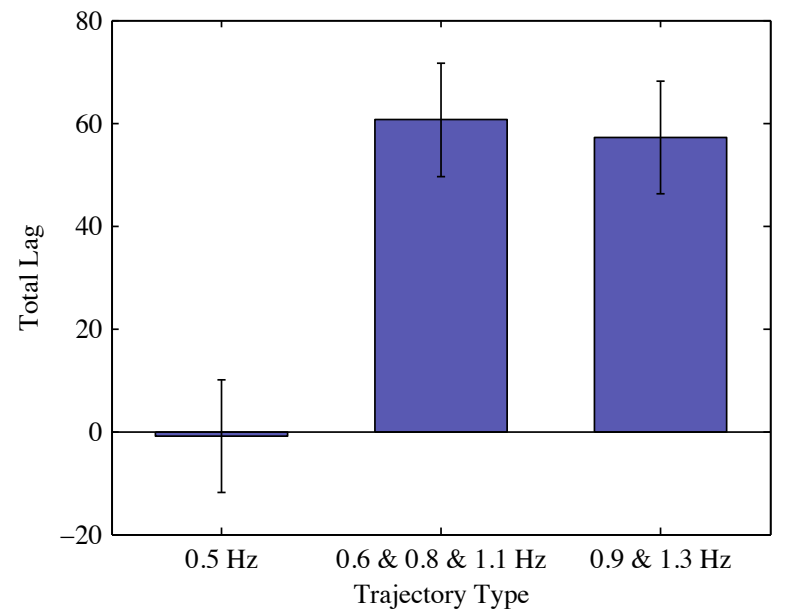

Fig. 6. Results of Total Lag vs Trajectory Type analysis between desired and recreated paths for fixed frequencies. A lower Total Lag indicates better tracking. Error bars represent $95 \%$ confidence interval.

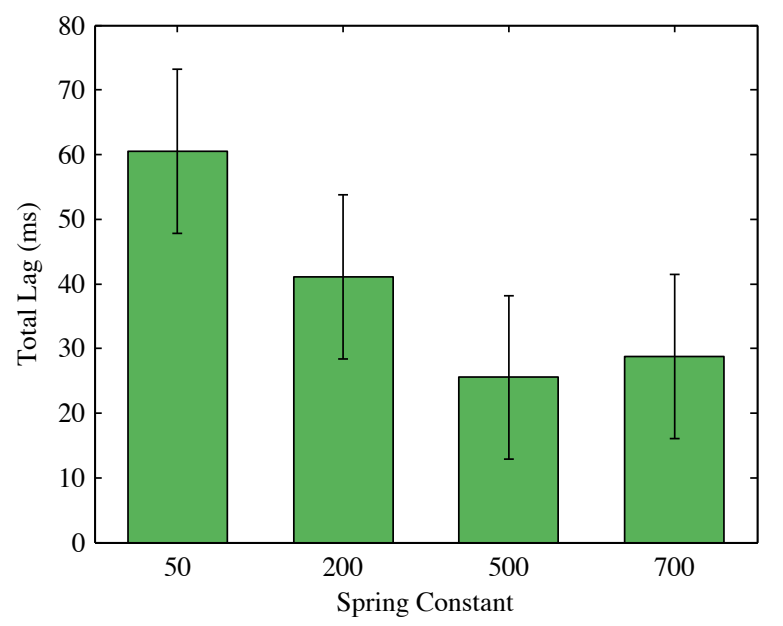

Fig. 7. Results of Total Lag analysis between actual and recreated paths vs Stiffness. A lower Total Lag indicates better tracking. Error bars represent $95 \%$ confidence interval.

a higher guiding stiffness results in better duplication of the efferent and afferent signals of each hand, and therefore may be preferred for bimanual rehabilitation.

The Total Lag for the chirp frequencies are shown in Fig. 8. Lower values indicate that the participants' output trajectory lagged the input less, and therefore shows better performance. There was a statistically significant difference $\left(p<10^{-4}\right)$ between symmetry modes. Post hoc analysis results are similar to those seen for the Power Score analysis of the fixed frequencies that show JSS is superior to PMS, however, VS is not distinguishable from either JSS or PMS. The Total Lag between the actual path of the guided hand and the recreated path was lower for JSS than the other symmetry modes. This indicates that JSS may result in a better duplication of the afferent signals and lead to faster relearning of the motor commands.

The results of the Average Lag analysis of the chirp frequency are shown in Fig. 9. Again, a lower lag is better. The Average Lag showed statistically significant differences

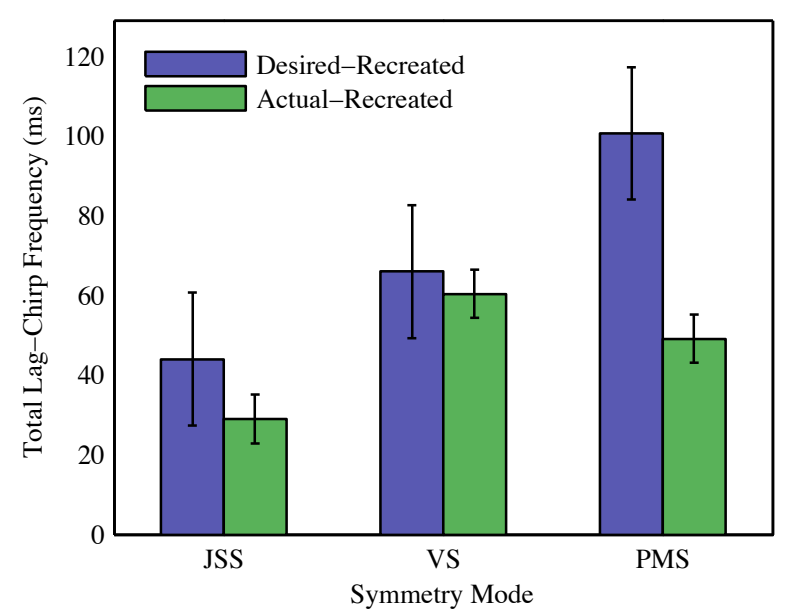

Fig. 8. Results of Total Lag analysis for chirp frequencies. Dark blue bars represent correlation between desired and recreated postion, light green bars indicate correlation between actual and recreated posion. Error bars represent $95 \%$ confidence interval.

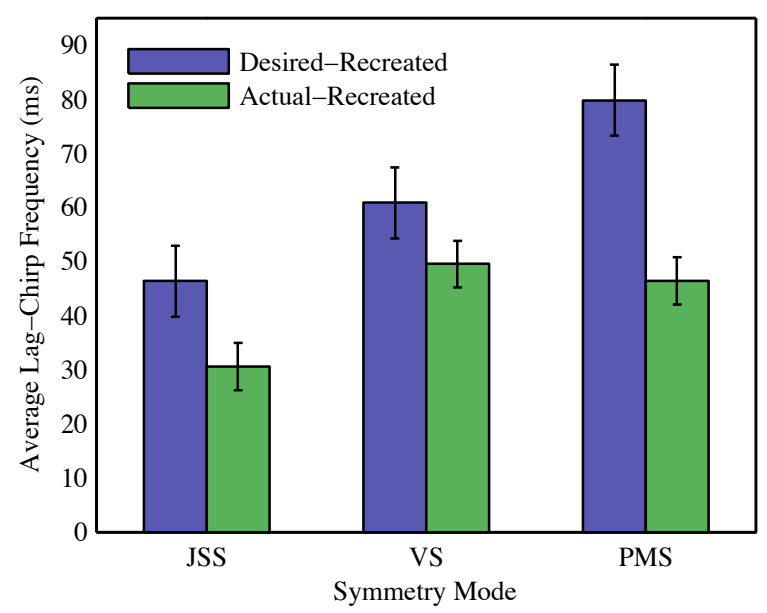

Fig. 9. Results of Average Lag analysis for chirp frequencies. Dark blue bars represent correlation between desired and recreated postion, light green bars indicate correlation between actual and recreated posion. Error bars represent $95 \%$ confidence interval.

$\left(p<10^{-4}\right)$ for the symmetry modes and for the virtual spring stiffnesses $\left(p<10^{-4}\right)$. Post hoc analysis showed that all three of the symmetry modes were statistically significantly different, with the best performance from JSS followed by VS and then PMS. Analysis of the Average Lag between the actual position of the guided hand and the recreated path showed the same results as the analysis of the Total Lag: the chirp frequencies were easier to reproduce in JSS.

Post hoc analysis of the virtual spring stiffness shows the same results as the Power Score of the fixed frequencies: the participant lagged more for the $50 \mathrm{~N} / \mathrm{m}$ spring stiffness and was, therefore, not following as well. Similar to the Power Score analysis, the Average Lag between the desired path and actual path of the guided hand for the $50 \mathrm{~N} / \mathrm{m}$ stiffness was greater than that of the higher stiffnesses.

For the chirp trials we also plotted an average of the lag as a function of time (frequency) for all participants for each combination of stiffness and symmetry mode. A representative 


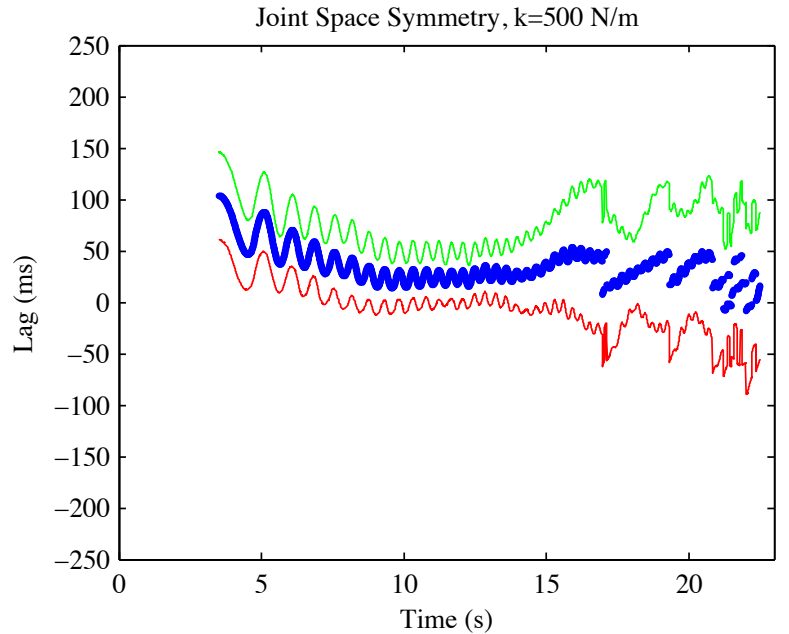

Fig. 10. Plot of Lag vs Time for all chirp trials (blue). The upper (green) and lower (red) bounds are set at one standard deviation.

plot can be seen in Fig. 10. Discontinuities in these plots were a result of at least one participant lagging enough to start leading the next motion. Most plots have an identifiable trend where the lag is increasing before a discontinuity occurs, and qualitatively, it was seen that this averaged lag was lower for JSS and at higher stiffnesses.

\section{CONCLUSions AND Future Work}

This study has addressed some of the questions regarding how symmetry modes and coupling stiffnesses affect tracking performance as it can be applied to bimanual rehabilitation trainers. We demonstrated that it was easier to reproduce motions in JSS and VSS symmetry modes than in the PMS mode. Additionally, in the previous study, we saw that VS motions were easier to follow [17]. However, we saw that for an increasing frequency chirp motion, JSS was easier to follow than VS or PMS. It was also interesting to note that towards the end of some chirp trials, the participant would resort to high frequency mirrored (JSS) motions. We hypothesize that this may be a result of the neurological and biomechanical advantages of JSS appearing during high frequency tasks, such as when clapping one's hands. We conclude that a combination of JSS and VSS symmetry modes may be preferred for bimanual rehabilitation.

These results also demonstrated that a guiding stiffness of $50 \mathrm{~N} / \mathrm{m}$ was difficult to follow and there was no significant difference between the other three guiding stiffnesses in terms of the recreated hand's ability to follow the desired path. The results do show that the stiffnesses less than $200 \mathrm{~N} / \mathrm{m}$ did not enable as much interlimb coordination as the higher stiffnesses. Since the goal is to have both hands move through a similar path, the lowest stiffness at or above $500 \mathrm{~N} / \mathrm{m}$ that an individual can use to perform the task is likely to be the most effective coupling stiffness for bimanual rehabilitation. The force will be large enough to ensure the afferent signals are duplicated, but soft enough that the impaired hand will still need to generate much of the force.
These results will guide the design of a bimanual rehabilitation trainer for home use that will have a mechanical linkage for selecting JSS, VS, or PMS symmetry and an adjustable spring to couple the hands. These results indicate that an adjustable spring setup would be preferable so the spring stiffness could easily be changed. This device will display the desired path visually and the participant will follow by moving their hands in a coordinated motion. The device will first be tested on healthy participants to refine the compliance stiffness and trajectories used. Home use trials will then be conducted on individuals with stroke to determine the device's ability to assist patients in regaining the use of their impaired limb.

\section{REFERENCES}

[1] L. Marchal-Crespo and D. Reinkensmeyer, "Review of control strategies for robotic movement training after neurologic injury," Journal of NeuroEngineering and Rehabilitation, vol. 6, no. 1, p. 20, 2009.

[2] G. Kwakkel, B. J. Kollen, and H. I. Krebs, "Effects of Robot-Assisted Therapy on Upper Limb Recovery After Stroke: A Systematic Review," Neurorehabil Neural Repair, vol. 22, no. 2, pp. 111-121, 2008.

[3] R. Oden, "Systematic therapeutic exercises in the management of the paralyses in hemiplegia," JAMA, vol. 23, pp. 828-833, 1918.

[4] J. Liepert, I. Uhde, S. Grf, O. Leidner, and C. Weiller, "Motor cortex plasticity during forced-use therapy in stroke patients: a preliminary study," Journal of Neurology, vol. 248, pp. 315-321, 2001.

[5] G. F. Wittenberg, R. Chen, K. Ishii, K. O. Bushara, E. Taub, L. H. Gerber, M. Hallett, and L. G. Cohen, "Constraint-Induced Therapy in Stroke: Magnetic-Stimulation Motor Maps and Cerebral Activation," Neurorehabil Neural Repair, vol. 17, no. 1, pp. 48-57, 2003.

[6] X. Feng, M. Johnson, L. Johnson, and J. Winters, "A suite of computerassisted techniques for assessing upper-extremity motor impairments,' in Conf Proc IEEE Eng Med Biol Soc, 2005.

[7] M. Johnson, X. Feng, L. Johnson, and J. Winters, "Potential of a suite of robot/computer-assisted motivating systems for personalized, home-based, stroke rehabilitation," Journal of NeuroEngineering and Rehabilitation, vol. 4, no. 1, p. 6, 2007.

[8] M. J. Johnson, H. F. M. Van der Loos, C. G. Burgar, P. Shor, and L. J. Leifer, "Experimental results using force-feedback cueing in robotassisted stroke therapy," IEEE Transactions on Neural Systems and Rehabilitation Engineering, vol. 13, pp. 335-348, 2005.

[9] M. Johnson, B. Ramachandran, R. Paranjape, and J. Kosasih, "Feasibility study of theradrive: a low-cost game-based environment for the delivery of upper arm stroke therapy," in Proc IEEE Eng Med Biol Soc, 2006.

[10] D. J. Reinkensmeyer, C. T. Pang, J. A. Nessler, and C. C. Painter, "Java therapy: Web-based robotic rehabilitation," Integration of Assistive Technology in the Information Age, vol. 9, pp. 66-71, 2001.

[11] C. Burgar, P. Lum, P. Shor, and H. Van der Loos, "Development of robots for rehabilitation therapy: The Palo Alto VA/Stanford experience," J. of Rehab Research and Development, vol. 37, pp. 663-674, 2000.

[12] S. L. Wolf, D. E. LeCraw, and L. A. Barton, "Comparison of Motor Copy and Targeted Biofeedback Training Techniques for Restitution of Upper Extremity Function Among Patients with Neurologic Disorders," Physical Therapy, vol. 69, no. 9, pp. 719-735, 1989.

[13] P. Lum, D. Reinkensmeyer, R. Mahoney, W. Z. Rymer, and C. Burgar, "Robotic devices for movement therapy after stroke: Current status and challenges to clinical acceptance," Topics in Stroke Rehab, vol. 8, pp. 40-53, 2002.

[14] S. Hesse, G. Schulte-Tigges, M. Konrad, A. Bardeleben, and C. Werner, "Robot-assisted arm trainer for the passive and active practice of bilateral forearm and wrist movements in hemiparetic subjects," Archives of Physical Medicine and Rehab, vol. 84, no. 6, pp. 915 - 920, 2003.

[15] J. Whitall, S. Waller, K. Silver, and R. Macko, "Repetitive Bilateral Arm Training With Rhythmic Auditory Cueing Improves Motor Function in Chronic Hemiparetic Stroke," Stroke, vol. 31, pp. 2390-2395, 2000.

[16] R. A. Schmidt and R. A. Bjork, "New conceptualizations of practice: Common principles in three paradigms suggest new concepts for training," Psychological Science, vol. 3, no. 4, pp. 207-217, 1992.

[17] H. G. Malabet, R. A. Robles, and K. B. Reed, "Symmetric motions for bimanual rehabilitation," in Proc. IEEE/RSJ Int Intelligent Robots and Systems (IROS) Conf, 2010, pp. 5133-5138. 